Compari son of the vel ocity di stribution bet ween the adhesi on appr oxi mat i on and the Eul er - Jeans- Newt on nodel

\begin{tabular}{|l|l|}
\hline 著者 & SOTAN Haj i me, TAKEKAWA Takayuki \\
\hline $\begin{array}{l}\text { j our nal or } \\
\text { publ i cat i on t i tl e }\end{array}$ & Physi cal r evi ew. [ Ser i es III . ] D \\
\hline vol une & 73 \\
\hline page r ange & 24024 \\
\hline year & $2006-01$ \\
\hline URL & ht t p: //hdl . handl e. net /10098/6419 \\
\hline
\end{tabular}




\title{
Comparison of the velocity distribution between the adhesion approximation and the Euler-Jeans-Newton model
}

\author{
Hajime Sotani ${ }^{1, *}$ and Takayuki Tatekawa ${ }^{1,2,3, \dagger}$ \\ ${ }^{1}$ Advanced Research Institute for Science and Engineering, Waseda University, 3-4-1 Okubo, Shinjuku, Tokyo 169-8555, Japan \\ ${ }^{2}$ Department of Physics, Ochanomizu University, 2-1-1 Otsuka, Bunkyo, Tokyo 112-8610, Japan \\ ${ }^{3}$ Department of Physics, Waseda University, 3-4-1 Okubo, Shinjuku, Tokyo 169-8555, Japan
}

(Received 19 December 2005; published 25 January 2006)

\begin{abstract}
For the evolution of density fluctuation in nonlinear cosmological dynamics, adhesion approximation (AA) is proposed as a phenomenological model, which is especially useful for describing nonlinear evolution. However, the origin of the artificial viscosity in AA is not clarified. Recently, Buchert and Domínguez report if the velocity dispersion of the dust fluid is regarded as isotropic, it works on a principle similar to viscosity or effective pressure, and they consider isotropic velocity dispersion as the origin of the artificial viscosity in AA. They name their model the Euler-Jeans-Newton (EJN) model. In this paper, we focus on the velocity distribution in AA and the EJN model and examine the time evolution in both models. We find the behavior of AA differs from that of the EJN model, i.e., although the peculiar velocity in the EJN model oscillates, that in AA is monotonically decelerated due to viscosity without oscillation. Therefore it is hard to regard viscosity in AA as effective pressure in the EJN model.
\end{abstract}

DOI: 10.1103/PhysRevD.73.024024

PACS numbers: 04.25.Nx, 95.30.Lz, 98.65.Dx

\section{INTRODUCTION}

The Lagrangian description for the cosmological fluid can be usefully applied to the structure formation scenario. This description provides a relatively accurate model even in a quasilinear regime. Zel'dovich [1] proposed a linear Lagrangian approximation for dust fluid. This approximation is called the Zel'dovich approximation (ZA) [1-10]. ZA describes the evolution of density fluctuation better than the Eulerian approximation [11-14]. Although ZA gives an accurate description until the quasilinear stage, ZA cannot describe the model after the formation of caustics. In ZA, even after the formation of caustics, the fluid elements keep moving in the direction set up by the initial condition.

In order to proceed with a hydrodynamical description in which caustics do not form, a qualitative pressure gradient [15] and thermal velocity scatter [3,16] in a collisionless matter have been discussed. Similarly, adhesion approximation (AA) [17] has been proposed, which is a model based on a nonlinear diffusion equation (i.e., Burgers's equation [18]). In AA, an artificial viscosity term is added to ZA; thus we can avoid caustics formation. The problem of structure formation has been discussed from the standpoint of AA [3,19-22], where it is shown that the density divergence does not occur in AA and that a density distribution close to the N-body simulation can be produced. However, the origin of the viscosity in AA has not yet been clarified.

Buchert and Domínguez [23] discussed the effect of velocity dispersion using the collisionless Boltzmann equation [24]. They argued that models of a large-scale

\footnotetext{
*Electronic address: sotani@gravity.phys.waseda.ac.jp

†Electronic address: tatekawa@ cosmos.phys.ocha.ac.jp
}

structure should be constructed by a flow describing the average motion of a multistream system. Then they showed that, when the velocity dispersion is regarded as small and isotropic, it produces effective pressure or viscosity terms, and they consider that the isotropic velocity dispersion corresponds to the origin of the artificial viscosity in AA. Furthermore, in consideration of kinematic theory, they derived the relation between mass density $\rho$ and pressure $P$, i.e., an equation of state. Buchert et al. [25] also showed how the viscosity term or the effective pressure of a fluid is generated, assuming that the peculiar acceleration is parallel to the peculiar velocity. Recently Buchert and Domínguez [26] provided an evaluation of the current status of adhesive gravitational clustering, which includes the above discussion, and they tried to improve past models. In their paper, they named their approach the EulerJeans-Newton (EJN) model. On the other hand, Domínguez $[27,28]$ proposed another approach. In these papers he clarified that a hydrodynamic formulation is obtained via a spatial coarse graining in a many-body gravitating system, and that the viscosity term in AA can be derived by the expansion of coarse-grained equations. This model is named the small-size expansion (SSE) model [26].

So far, with respect to the correspondence of the viscosity term with the effective pressure, and with regard to the extension of the Lagrangian description to various matter, the EJN model has been considered. Actually, Adler and Buchert [29] have formulated the Lagrangian perturbation theory for a barotropic fluid. Morita and Tatekawa [30], Tatekawa et al. [31], and Tatekawa [32,33] solved the Lagrangian perturbation equations for a polytropic fluid. However, it is still an open problem whether AA could realize the behavior of the EJN model in the proper way. It is known that the viscosity in AA decelerates the peculiar 
velocity in a dense region and avoids the formation of the caustic, while the effective pressure in the EJN model also decelerates the motion of the fluid. But the fluid in the EJN model not only would decelerate but also might bounce in a dense region due to the effective pressure, which is known as the Jeans instability for cosmological fluid. In other words, we consider that AA would not realize the behavior of the EJN model.

In this paper, to compare AA and the EJN model, we especially analyze the peculiar velocity in a cylindrical collapse model. Although we already compared AA with the EJN model with respect to density fluctuation in a past paper [34], because we used an explicit method for solving partial differential equations, the accuracy of the numerical calculation in the dense region was not good. Therefore we could not observe the Jeans instability but, rather, the numerical instability. In this paper, instead of the explicit method, we apply the iterated Crank-Nicholson method [35]. This method resolves the difficulty of solving the resulting implicit algebraic equations in the original Crank-Nicholson method and preserves the good stability properties.

As our analyses show, although the peculiar velocity in AA decelerates due to the viscosity, the velocity does not oscillate. On the other hand, as a preliminary expectation, the peculiar velocity in the EJN model decelerates and also oscillates. Although the tendency of the evolution of the peculiar velocity would depend on the viscous parameter or the Jeans length, the behavior of AA is obviously different from that of the EJN model. We notice that, while both AA and the EJN model certainly describe the quasinonlinear evolution well, the detail of the evolution is different. When we take a large value for the Jeans length, the fluid bounces to the outside. On the other hand, when we take a small value for the Jeans length, although the collapse of the cylindrical matter decelerates, a caustic might finally form at the center. Therefore it is problematic that the viscous term in AA is explained as an effect similar to the effective pressure term in the EJN model. For an explanation of the viscous term in AA, we will have to analyze more Lagrangian models.

This paper is organized as follows. In Sec. II, we present Lagrangian perturbative solutions in the Einstein-de Sitter (E-dS) universe. First, we show linear perturbative solutions for dust fluid in Sec. II A, and in Sec. II B we mention the problem of ZA and show the solution of AA. Then we explain the EJN model in Sec. II C. In Sec. III we compare the evolution of the peculiar velocity in AA with that in the EJN model. Finally in Sec. IV we discuss our results and state our conclusions.

\section{THE LAGRANGIAN DESCRIPTION FOR THE COSMOLOGICAL FLUID}

In this section, we briefly present perturbative solutions in the Lagrangian description. In Newtonian cosmology, to introduce cosmic expansion, we adopt the coordinate transformation from physical coordinates $\boldsymbol{r}$ to comoving coordinates $\boldsymbol{x}$, such as $\boldsymbol{x}=\boldsymbol{r} / a(t)$, where $a(t)$ is a scale factor. In Lagrangian hydrodynamics, the comoving coordinates $\boldsymbol{x}$ of the fluid elements are represented in terms of Lagrangian coordinates $\boldsymbol{q}$ as

$$
\boldsymbol{r}=a \boldsymbol{x}=a(\boldsymbol{q}+\boldsymbol{s}(\boldsymbol{q}, t)),
$$

where $\boldsymbol{s}$ denotes the Lagrangian displacement vector due to the presence of inhomogeneities. With the Jacobian of the coordinate transformation from $\boldsymbol{x}$ to $\boldsymbol{q}, \quad J \equiv$ $\operatorname{det}\left(\partial x_{i} / \partial q_{j}\right)=\operatorname{det}\left(\delta_{i j}+\partial s_{i} / \partial q_{j}\right)$, the mass density is described exactly as

$$
\rho=\rho_{b} J^{-1},
$$

where $\rho_{b}$ means background average density. Furthermore we can decompose $s$ into the longitudinal and the transverse modes, i.e., $s=\nabla_{q} S+S^{T}$ with $\nabla_{q} \cdot S^{T}=0$. In this paper, we consider only the longitudinal mode for simplicity. The evolution equation for the longitudinal mode is written as follows $[29,30]$ :

$$
\nabla_{x}\left(\ddot{\boldsymbol{s}}+2 \frac{\dot{a}}{a} \dot{\boldsymbol{s}}-\frac{\kappa \gamma \rho_{b}^{\gamma-1}}{a^{2}} J^{-\gamma} \nabla_{x} J\right)=-4 \pi G \rho_{b}\left(J^{-1}-1\right),
$$

where the dot above the variables denotes the partial derivative with respect to $t$. In general, it is very difficult to solve this equation for such reasons as the coordinate transformation or nonlocality. In order to avoid this difficulty, we apply the perturbative approach and impose symmetry in Eq. (3). Particularly in this paper we consider cylindrical symmetry, and the evolution equation in a cylindrical-symmetric model is given in the appendix.

\section{A. The Lagrangian perturbation for dust fluid}

Zel'dovich derived a first-order solution of the longitudinal mode for dust fluid [1]. In the Friedmann Universe model, the solutions are formally written as follows:

$$
S(\boldsymbol{q}, t)=D_{+}(t) S_{+}(\boldsymbol{q})+D_{-}(t) S_{-}(\boldsymbol{q}),
$$

where $D_{+}(t)$ and $D_{-}(t)$ mean the growing factor and the decaying factor, respectively. This first-order approximation is called the Zel'dovich approximation (ZA). In the case of the Einstein-de Sitter Universe, $D_{+}(t)$ and $D_{-}(t)$ are described as

$$
D_{+}(t) \propto t^{2 / 3}
$$

$$
D_{-}(t) \propto t^{-1} .
$$

Especially when we consider the plane-symmetric case, ZA gives exact solutions [2,36]. 


\section{B. Adhesion approximation (AA)}

Although the Lagrangian approximation gives an accurate description until a quasilinear regime develops, it cannot describe the model after the formation of caustics. After that, the nonlinear structure diffuses because the fluid elements keep moving in the direction set up by the initial condition. In order to avoid caustics formation, the adhesion approximation (AA) [17] was proposed, which is a model based on a nonlinear diffusion equation (Burgers's equation [18]). In AA, an artificial viscosity term is added to ZA. In ZA, the equation for "peculiar velocity" $\boldsymbol{u}$ is written as follows:

$$
\begin{gathered}
\frac{\partial \boldsymbol{u}}{\partial D_{+}}+\left(\boldsymbol{u} \cdot \nabla_{x}\right) \boldsymbol{u}=0, \\
\boldsymbol{u} \equiv \frac{\partial \boldsymbol{x}}{\partial D_{+}}=\frac{\dot{\boldsymbol{x}}}{\dot{D_{+}}},
\end{gathered}
$$

where $D_{+}$is the growing factor in ZA. To avoid caustics formation, in AA we add an artificial viscosity term to the right side of Eq. (7), i.e.,

$$
\frac{\partial \boldsymbol{u}}{\partial D_{+}}+\left(\boldsymbol{u} \cdot \nabla_{x}\right) \boldsymbol{u}=\nu \nabla_{x}^{2} \boldsymbol{u} .
$$

Now we introduce the Hopf-Cole transformation [37,38] such as

$$
\boldsymbol{u}=-\nu \nabla_{x}\left(\log \theta\left(\boldsymbol{x}, D_{+}\right)\right)
$$

then Eq. (9) is changed to a diffusion equation:

$$
\frac{\partial \theta}{\partial D_{+}}=\nu \nabla_{x}^{2} \theta
$$

Meanwhile, by using the inverse Hopf-Cole transformation, we obtain the solution of Eq. (9):

$$
\begin{gathered}
\boldsymbol{x}=\boldsymbol{q}+\int_{D_{0}}^{D_{+}} \boldsymbol{u}\left(\boldsymbol{x}\left(\boldsymbol{q}, D^{\prime}\right), D^{\prime}\right) d D^{\prime}, \\
\boldsymbol{u}=\frac{\int d^{3} \boldsymbol{x}^{\prime} \frac{\left(\boldsymbol{x}-\boldsymbol{x}^{\prime}\right)}{D} G\left(\boldsymbol{x}, \boldsymbol{x}^{\prime}\right)}{\int d^{3} \boldsymbol{x}^{\prime} G\left(\boldsymbol{x}, \boldsymbol{x}^{\prime}\right)} \\
G\left(\boldsymbol{x}, \boldsymbol{x}^{\prime}\right)=\exp \left[-\frac{1}{2 \nu}\left(\Psi_{0}\left(\boldsymbol{x}^{\prime}\right)+\frac{\left(\boldsymbol{x}-\boldsymbol{x}^{\prime}\right)^{2}}{2 D}\right)\right],
\end{gathered}
$$

where

$$
\nabla_{x} \Psi_{0}(\boldsymbol{x}) \equiv \boldsymbol{s}_{0} .
$$

We consider the case when the viscosity coefficient is quite small $[\nu \rightarrow+0(\nu \neq 0)]$. Within the limits of a small $\nu$, the analytic solution of Eq. (9) is given by

$$
\boldsymbol{u}(\boldsymbol{x}, t)=\frac{\sum_{\alpha}\left(\frac{\boldsymbol{x}-\boldsymbol{q}_{\alpha}}{D_{+}}\right) j_{\alpha} \exp \left(-\frac{I_{\alpha}}{2 \nu}\right)}{\sum_{\alpha} j_{\alpha} \exp \left(-\frac{I_{\alpha}}{2 \nu}\right)},
$$

where $\boldsymbol{q}_{\alpha}$ is the Lagrangian points that minimize the action

$$
\begin{gathered}
I_{\alpha} \equiv I\left(\boldsymbol{x}, a ; \boldsymbol{q}_{\alpha}\right)=S_{0}\left(\boldsymbol{q}_{\alpha}\right)+\frac{\left(\boldsymbol{x}-\boldsymbol{q}_{\alpha}\right)^{2}}{2 a}=\min , \\
\left.j_{\alpha} \equiv\left[\operatorname{det}\left(\delta_{i j}+\frac{\partial^{2} S_{0}}{\partial q_{i} \partial q_{j}}\right)\right]^{-1 / 2}\right|_{\boldsymbol{q}=\boldsymbol{q}_{\alpha}}, \\
S_{0}=S\left(\boldsymbol{q}, t_{0}\right),
\end{gathered}
$$

considered as a function of $\boldsymbol{q}$ for fixed $\boldsymbol{x}$ [21], where the Roman character index denotes the Cartesian coordinate. In this paper, we consider the cylindrical-symmetric case. For this case, we have to change the evolution equation, Eq. (9), slightly (see Sec. III A).

\section{The EJN model}

Although AA seems a good model for avoiding the formation of caustics, the origin of the modification (or artificial viscosity) is not clarified. Buchert and Domínguez [23] argued that the effect of velocity dispersion is important in hindering caustics formation. They showed that, when the velocity dispersion is still small and can be regarded as isotropic, it behaves as effective pressure or viscosity terms. Under the consideration of fluid kinematics, they proposed the effective equation of state as $p \propto$ $\rho^{5 / 3}$. Also Buchert et al. [25] showed how the viscosity term is generated by the effective pressure of a fluid under the assumption that the peculiar acceleration is parallel to the peculiar velocity. Moreover, Buchert and Domínguez [26] recently provided an evaluation of the current status of adhesive gravitational clustering, which is included in the above discussion. In their paper, they named their approach the Euler-Jeans-Newton (EJN) model.

When we consider the polytropic equation of state $P=$ $\kappa \rho^{\gamma}$, the first-order solutions for the longitudinal mode can be written in Fourier space as follows [30]: for $\gamma \neq 4 / 3$,

$$
\hat{S}(\boldsymbol{K}, a) \propto a^{-1 / 4} J_{ \pm 5 /(8-6 \gamma)}\left(\sqrt{\frac{2 C_{2}}{C_{1}}} \frac{|\boldsymbol{K}|}{|4-3 \gamma|} a^{(4-3 \gamma) / 2}\right),
$$

where $\hat{S}$ is the Fourier transformation of $S, \boldsymbol{K}$ is Lagrangian wave number, and $J_{\nu}$ denotes the Bessel function of order $\nu$, and for $\gamma=4 / 3$,

$$
\hat{S}(\boldsymbol{K}, a) \propto a^{-1 / 4 \pm \sqrt{25 / 16-C_{2}|\boldsymbol{K}|^{2} / 2 C_{1}},}
$$

where $C_{1} \equiv 4 \pi G \rho_{b}\left(a_{\text {in }}\right) a_{\text {in }}^{3} / 3, C_{2} \equiv \kappa \gamma \rho_{b}\left(a_{\text {in }}\right)^{\gamma-1} a_{\text {in }}^{3(\gamma-1)}$, and $a_{\text {in }}$ means the scale factor given as an initial condition. When we take the limit $\kappa \rightarrow 0$, these solutions agree with Eq. (4).

In this model, the behavior of the solutions strongly depends on the relation between the scale of fluctuation and the Jeans scale. Here we define the Jeans wave number as 


$$
K_{\mathrm{J}} \equiv\left(\frac{4 \pi G \rho_{b} a^{2}}{d P / d \rho\left(\rho_{b}\right)}\right)^{1 / 2} .
$$

The Jeans wave number, which gives a criterion for whether a density perturbation with a wave number will grow or decay with oscillation, depends on time in general. If the polytropic index $\gamma$ is smaller than $4 / 3$, all modes become decaying modes and the fluctuation will disappear. On the other hand, if $\gamma>4 / 3$, all density perturbations will grow to collapse. In the case where $\gamma=4 / 3$, the growing and decaying modes coexist at all times. We can rewrite the first-order solution Eq. (20) with the Jeans wave number, i.e.,

$$
\hat{S}(\boldsymbol{K}, a) \propto a^{-1 / 4} J_{ \pm 5 /(8-6 \gamma)}\left(\frac{\sqrt{6}}{|4-3 \gamma|} \frac{|\boldsymbol{K}|}{K_{\mathrm{J}}}\right) .
$$

In this paper, we analyze the first-order perturbation. When we consider cylindrical-symmetric models, even if we deal with only the first-order perturbation, they cannot be analyzed in Fourier space, and we need to solve partial differential equations in real space with a numerical method (also see the appendix).

\section{TIME EVOLUTION IN CYLINDRICAL MODEL}

For the cylindrical-symmetric case, dust collapse has been analyzed [14]. Here we consider the evolution with $\mathrm{ZA}$, AA, and the EJN models in the E-dS Universe. Hereafter, we define $R$ and $r$ as Eulerian and Lagrangian radial coordinates, respectively. At the initial time, we can identify the Lagrangian coordinate with the Eulerian one, i.e., $R=r$. Previous studies considered the collapse and/or evolution with the top-hat density distribution as the initial condition. Although the evolution of this model is easy to compute, the boundary condition becomes discontinuous. To avoid a discontinuity of the pressure gradient, we adopt the Mexican-hat-type model (Fig. 1):

$$
\delta(R)=\varepsilon\left(2-R^{2}\right) e^{-R^{2} / 2},
$$

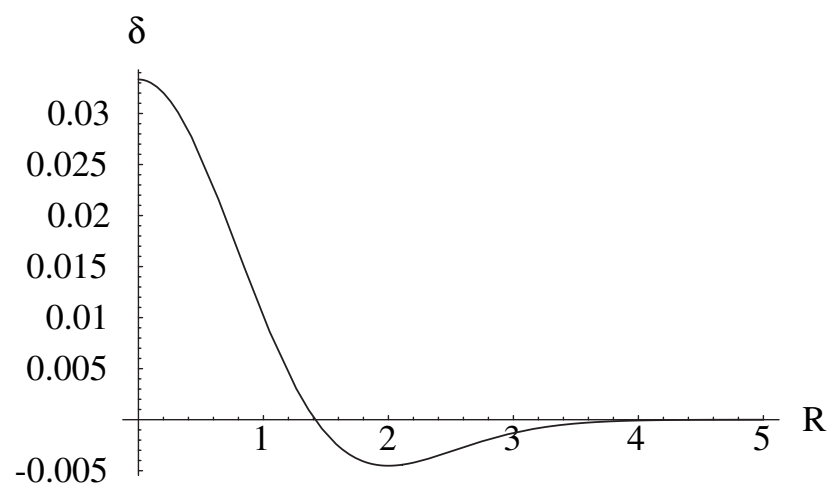

FIG. 1. Mexican-hat-type model. The average of density fluctuation over the whole space becomes zero. where $R$ is the Eulerian comoving radial coordinate. This model has several merits, for example, the fluctuation is derived by the 2 times differential calculus of Gaussian, i.e.,

$$
\begin{aligned}
-\nabla^{2}\left(\varepsilon e^{-R^{2} / 2}\right) & =-\frac{1}{R} \frac{\partial}{\partial R}\left(R \frac{\partial}{\partial R}\left(\varepsilon e^{-R^{2} / 2}\right)\right) \\
& =\varepsilon\left(2-R^{2}\right) e^{-R^{2} / 2},
\end{aligned}
$$

and the average of density fluctuation over the whole space becomes zero:

$$
\int_{0}^{\infty} 2 \pi R \delta(R) d R=0 .
$$

For this model, from Eq. (4) the solution of ZA is given as follows:

$$
S(a, r)=-a \varepsilon e^{-r^{2} / 2} .
$$

In our analysis, we set $\varepsilon=1$ due to an advantage of linear analysis, and the initial scale factor is set $a_{0}=0.0167(=$ $1 / 60)$ as the initial condition, where the initial density fluctuation at $r=0$ becomes $\delta=1 / 30$ and the caustic appears at $a=1$. On the other hand, for AA and the EJN model we can determine the initial longitudinal mode $S$ and the initial peculiar velocity by preference as the initial conditions. In this paper they are made equal with those of the growing mode in ZA. Thus, the initial conditions for $\mathrm{AA}$ and the EJN model are given by

$$
\begin{gathered}
S\left(a_{0}, r\right)=-a_{0} \varepsilon e^{-r^{2} / 2}, \\
\left.\frac{\partial S(a, r)}{\partial a}\right|_{a=a_{0}}=-\varepsilon e^{-r^{2} / 2} .
\end{gathered}
$$

\section{A. The adhesion approximation}

First we consider the evolution in the AA model. The evolution of the fluctuation is described by Eq. (9). For the cylindrical case, we slightly change the evolution equation. When we introduce cylindrical coordinates and assume the cylindrical symmetry, Burgers's equation is described as follows [39]:

$$
\partial_{\tau} u+u \partial_{\chi} u=\nu\left[\frac{1}{\chi} \partial_{\chi}\left(\chi \partial_{\chi} u\right)-\frac{u}{\chi^{2}}\right]=\nu \partial_{\chi}\left(\frac{1}{\chi} \partial_{\chi}(\chi u)\right)
$$

where $\tau$ and $\chi$ are the time variable and the radial coordinate, respectively. Under a transformation such as

$$
u=-\frac{2 \nu}{\theta} \frac{\partial \theta}{\partial \chi}
$$

Eq. (30) is rewritten as

$$
\partial_{\tau} \theta=\frac{\nu}{\chi} \partial_{\chi}\left(\chi \partial_{\chi} \theta\right) .
$$

The generic solution for Eq. (32) is described by the 
integral form:

$$
\begin{gathered}
\theta(\chi, \tau)=k(\tau) \exp \left(-\frac{1}{2 \nu} \int_{0}^{\chi} u(\omega, \tau) d \omega\right), \\
k(\tau)=\theta(0, \tau) \\
\theta(0, \tau)=\theta_{0}(\chi)=k(0) \exp \left(-\frac{1}{2 \nu} \int_{0}^{\chi} u(\omega, \tau) d \omega\right) .
\end{gathered}
$$

In this paper we apply the cylindrical Burgers's equation (30) for the adhesion model. In the E-dS Universe model, the evolution equation is given as

$$
\frac{\partial u}{\partial a}+u \frac{\partial u}{\partial R}=\nu \frac{\partial}{\partial R}\left[\frac{1}{R} \frac{\partial}{\partial R}(R u)\right]
$$

Now we introduce Lagrangian displacement for the radial coordinate from Eq. (1):

$$
R=r+\partial_{r} S(r, t),
$$

where $R$ and $r$ mean Eulerian and Lagrangian radial coordinate, respectively, and $S$ means Lagrangian displacement potential. Because we assume cylindrical symmetry and irrotational motion, the Lagrangian perturbation includes only the longitudinal mode. Also the peculiar velocity [Eq. (8)] is rewritten as

$$
u=\frac{\partial}{\partial a}\left(\frac{\partial S}{\partial r}\right)
$$

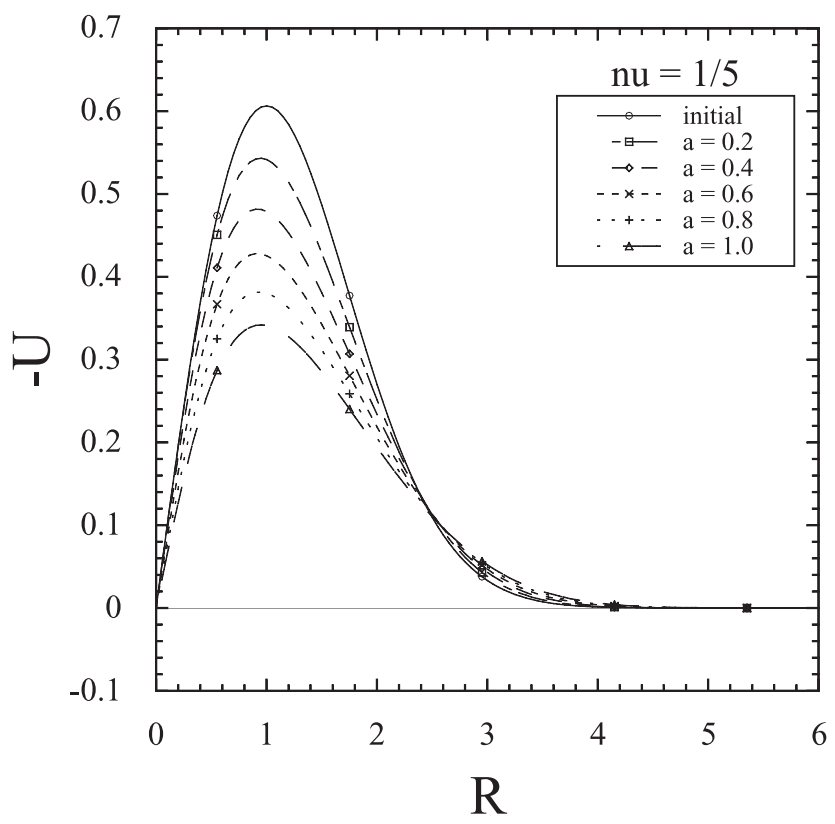

FIG. 2. The evolution of the peculiar velocity in the AA model. Here we show the velocity distribution at $a=1 / 60$ (initial, solid line) and $a=0.2,0.4, \ldots, 1.0$. During evolution, the velocity decelerates because the viscosity resists the motion of the fluid. In AA, although the velocity decelerates, it does not oscillate.
Using this peculiar velocity, the evolution equation of the adhesion model is described by Eq. (36), and we examine the evolution of the peculiar velocity. For comparison, we calculate cylindrical models with the viscous parameter $\nu$. Figure 2 shows the evolution of the peculiar velocity with $\nu=1 / 5$, where we show the relation between Eulerian coordinates $R$ and the peculiar velocity $U$. If we take a limit $\nu \rightarrow 0$, i.e., for the case of dust (ZA), the distribution of the peculiar velocity at any given time is the same as the initial distribution. On the other hand, from Fig. 2 we can see that the peculiar velocity decelerates monotonically due to the viscosity.

We notice that Eq. (36) is described with Eulerian comoving coordinates. Thus it is complicated in a degree if we obtain the Lagrangian displacement [cf. Eq. (12)]. In fact, for evolution of the fluctuation we need to consider the correspondence between Eulerian coordinates $\boldsymbol{x}$ and Lagrangian coordinates $\boldsymbol{q}$ for every grid at every time step.

\section{B. The EJN model}

Next, we analyze evolution in the EJN model. If we consider a cylindrical model, because of mode coupling in Laplacian, we cannot separate the perturbation to the spacial-dependent and the time-dependent term. Therefore we adopt numerical calculation for the evolution of the EJN model. In a previous paper [34], we apply an explicit method for solving partial differential equations [40]. However, this method tends to produce numerical instability. To avoid the numerical instability, we adopt another method. Teukolsky [35] discussed the iterated Crank-Nicholson method, which is one implicit scheme for numerical calculation. The method resolves the difficulty of solving the resulting implicit algebraic equations in the original Crank-Nicholson method and preserves the good stability properties.

The peculiar velocity in comoving coordinates is described as

$$
\boldsymbol{v}(a, \boldsymbol{q}) \equiv \frac{\partial}{\partial a}\left(\nabla_{q} S(a, \boldsymbol{q})\right) .
$$

Because we consider a cylindrical-symmetric model, we compute only radial velocity, i.e.,

$$
\boldsymbol{V}_{r}(a, r) \equiv \frac{\partial}{\partial a}\left(\frac{\partial S(a, r)}{\partial r}\right)
$$

where $r$ is the Lagrangian radial coordinate. From the definition of the peculiar velocity, we notice it does not change in the dust model (ZA). Because the solution in the dust model can be decomposed in time and spacial components, the peculiar velocity at any time is the same as the initial peculiar velocity. Furthermore, the growing factor of the dust model is given by Eq. (5). Therefore the longitudinal mode $S$ in ZA monotonously increases [Eq. (27)].

Correspondingly, we analyze the peculiar velocity in the EJN model. For the numerical calculation, we set the 
boundary condition at $r=0$ and $r=10$ such as

$$
\begin{gathered}
\left.\frac{\partial S(a, r)}{\partial r}\right|_{r=0}=0, \\
S(a, 10)=\left.\frac{\partial S(a, r)}{\partial r}\right|_{r=10}=0 .
\end{gathered}
$$

Because the distribution is asymptotically homogeneous, we set that the fluid does not move at the outside. The behavior of the EJN model strongly depends on the parameters $\kappa$ and $\gamma$. If $\kappa$ is very small, the solution is similar to that in ZA. On the other hand, if $\kappa$ is very large, the fluctuation oscillates and disappears. Following our previous method [34], we choose a reasonable value of the parameter $\gamma$, i.e., $\gamma=4 / 3$ and $5 / 3$. In the case of $\gamma=$ $4 / 3$, as we showed in Eq. (21), the solutions can be described simply and have both growing and decaying modes. In the case of $\gamma=5 / 3$, Buchert and Domínguez [23] claimed that the isotropic velocity dispersion corresponds to the origin of the artificial viscosity in AA. Further, instead of $\kappa$, we set the value of the Jeans wave number $K_{\mathrm{J}}$ [Eq. (22)].

Figures 3 and 4 show the peculiar velocity in the EJN model with linear approximation. We can see that the solution has both growing and decaying modes in the early stage, and that the position where the peculiar velocity is

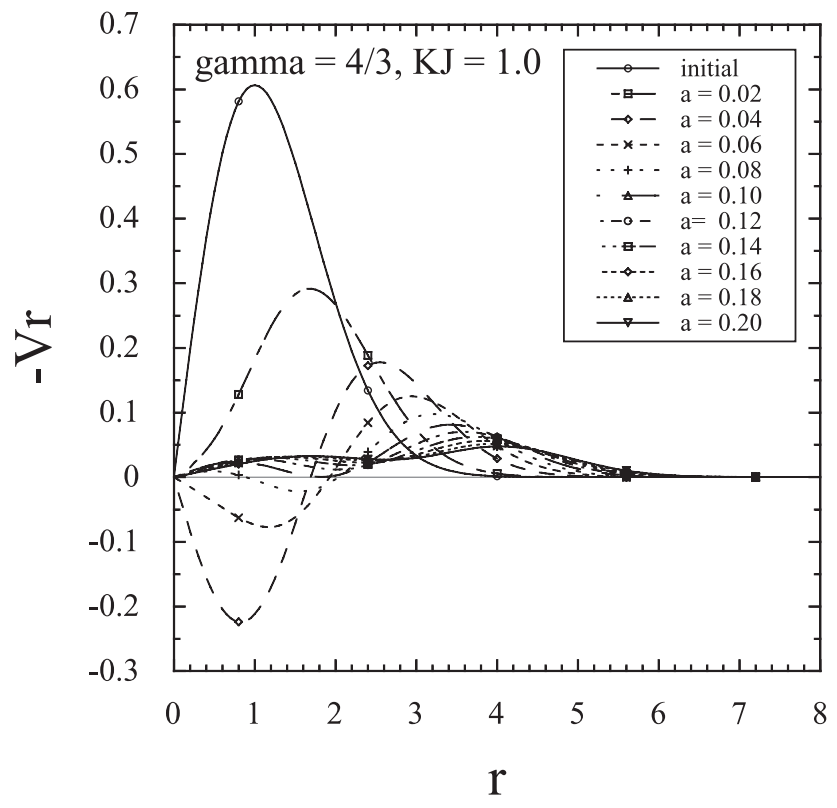

FIG. 3. The evolution of the peculiar velocity in the EJN model $\left(\gamma=4 / 3, K_{J}=1.0\right)$. We show the time slices at $a=$ $0.02,0.04, \ldots, 0.2$. We also show the velocity distribution at $a=$ $1 / 60$ (initial, solid line). During time evolution, the peak of the peculiar velocity moves to the outside. Because of the effect of the pressure, the form of the distribution of the peculiar velocity changes from the initial one. The velocity decelerates and the falling fluid at the initial time bounces to the outside.

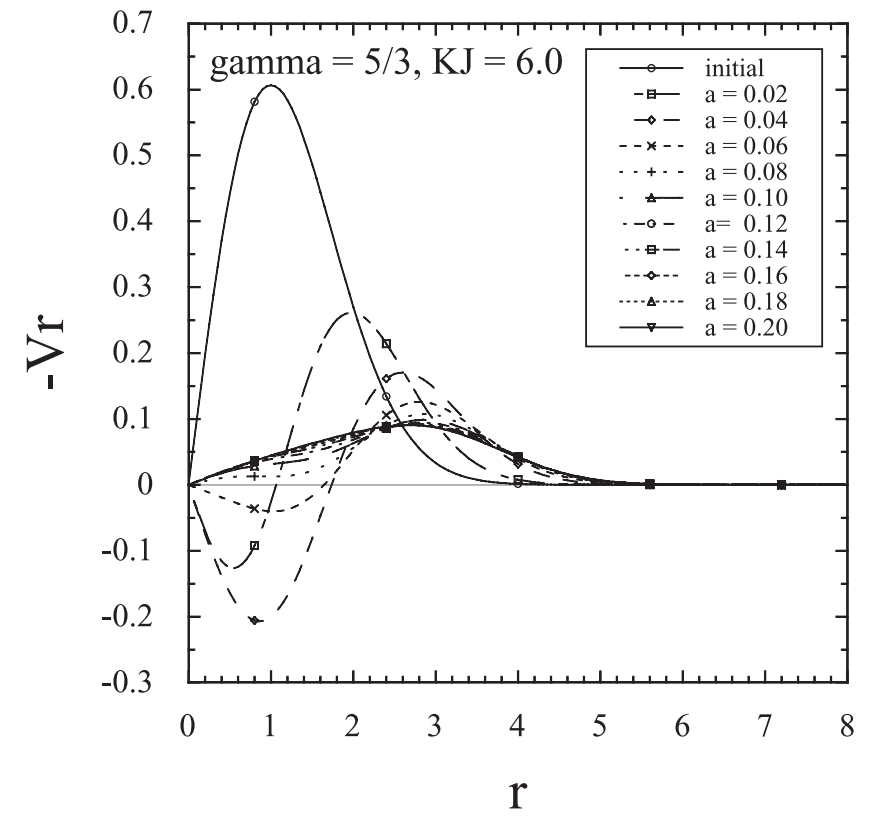

FIG. 4. The evolution of the peculiar velocity in the EJN model $\left(\gamma=5 / 3, K_{J}=6.0\right)$. We show the time slices at $a=$ $0.02,0.04, \ldots, 0.2$. We also show the velocity distribution at $a=$ $1 / 60$ (initial, solid line). Similar to the case where $\gamma=4 / 3$, during time evolution, the peak of the peculiar velocity moves to the outside. Because of the effect of the pressure, the distribution of the peculiar velocity oscillates at $0.02<a<0.2$.

the fastest moves outward. Then the distribution of the peculiar velocity changes by the effect of the pressure. Moreover, it is known that in Cartesian coordinates the solution for $\gamma \geq 4 / 3$ always has a growing mode, and that the peculiar velocity is not zero at any time (especially in the case where $\gamma=5 / 3$ the perturbative solutions asymptotically approach those of the dust model). Therefore as seen Figs. 3 and 4, the distribution of the peculiar velocity slightly oscillates, and the peak of the peculiar velocity moves to the outside at the early stage. Then, finally, caustics would form in the EJN model as in the case of the dust model (ZA).

\section{DISCUSSION AND CONCLUDING REMARKS}

With respect to the distribution of the peculiar velocity, we examine the correspondence between AA and the EJN model with cylindrical symmetry. In this analysis, even if we consider linear perturbation, it is hard to describe the solution with explicit form. Therefore we carried out numerical calculation, where to avoid numerical instability we adopted the iterated Crank-Nicholson method. From our calculation, when we take small value for the Jeans wave number, the peculiar velocity in the EJN model oscillates due to the pressure. For the cosmological fluid, such oscillation is known as the Jeans instability. On the other hand, the peculiar velocity in AA is decelerated due 
to the viscosity, where there is no oscillation. Thus we can see that the behavior of AA is different from that of the EJN model.

Furthermore, when we take a large value for the Jeans wave number in the EJN model, the evolution of the peculiar velocity is similar to that in AA (see Fig. 5). However, it is well known that in Cartesian coordinates the linear perturbative solutions for $\gamma \geq 4 / 3$ in the EJN model have a growing solution. Therefore the perturbation in the EJN model would eventually diverge. In other words, we can predict that, even if we take a large value for the Jeans wave number in the EJN model, the radial motion of the fluid does not stop and finally collapses. Thus, there also exists an essential difference between AA and the EJN model on this point, because it is known that the formation of caustic does not occur in AA. Hence, AA cannot express the EJN model with propriety and, in order to clarify the origin of the viscosity term in AA, we should consider other effects besides isotropic velocity dispersion or isotropic effective pressure.

Recently, Buchert and Domínguez [26] discussed adhesive gravitational clustering and tried to provide a clear explanation of the assumptions for adhesion approximation. They applied the Eulerian and Lagrangian expansions to the nonperturbative regime and proposed a new nonperturbative approximation. When we analyze this new approach with both analytic and numerical methods, we

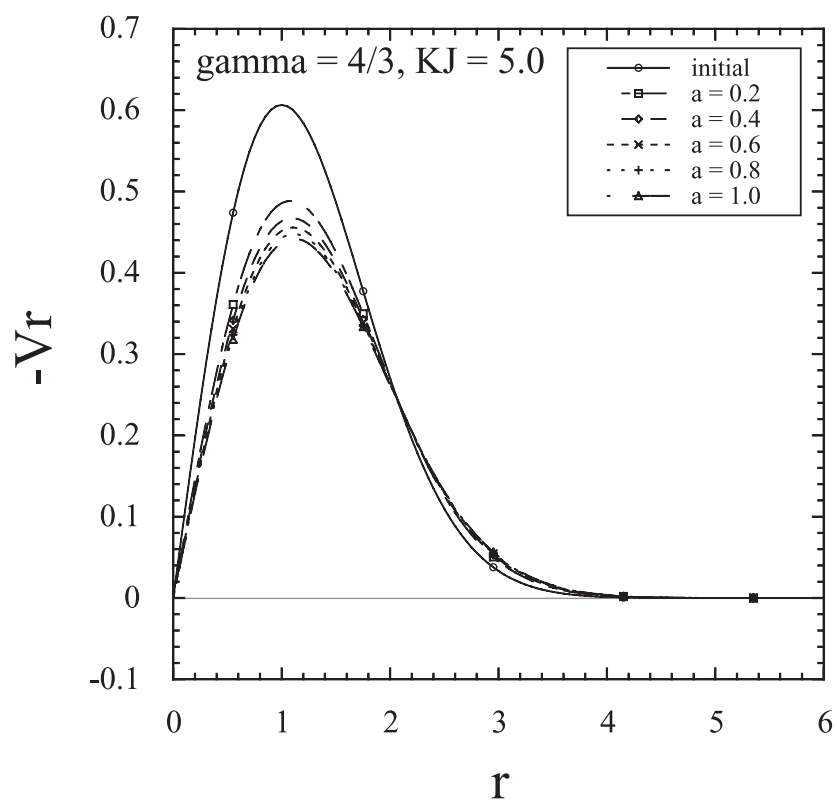

FIG. 5. The evolution of the peculiar velocity in the EJN model $\left(\gamma=4 / 3, K_{J}=5.0\right)$. We show the time slices at $a=$ $0.2,0.4, \ldots, 1.0$. We also show the velocity distribution at $a=$ $1 / 60$ (initial, solid line). During time evolution, the peak of the peculiar velocity slightly moves to the outside. In this case, the peculiar velocity does not oscillate. However, the cylindrical dust would collapse, because the deceleration of the peculiar velocity is gentle. may be able to explicate the origin of the artificial viscosity in AA. Also, as a future work, we would describe the evolution of the density fluctuation in a nonlinear regime with a semianalytic method.

\section{ACKNOWLEDGMENTS}

We thank Masahiro Morikawa for useful discussion. T. T. thanks the Mathematics Library, Hokkaido University, for pointing out some references. This work was supported by the Grant-in-Aid for Scientific Research Fund of the Ministry of Education, Culture, Sports, Science and Technology, Japan (Young Scientists (B) 16740152).

\section{APPENDIX: THE EVOLUTION EQUATION IN CYLINDRICAL MODEL}

In Sec. IIC, we noticed that Eq. (3) is hard to solve because of the coordinate transformation or nonlocality. In this appendix we rewrite the equation with cylindrical symmetry. We can introduce Lagrangian displacement for the radial coordinate by Eq. (37). The spacial derivative is rewritten with the Lagrangian coordinate, such as

$$
\frac{\partial}{\partial r}=\frac{\partial R}{\partial r} \frac{\partial}{\partial R}=\left(1+\partial_{r}^{2} S\right) \frac{\partial}{\partial R} \text {. }
$$

Therefore, the derivative is changed as

$$
\frac{\partial}{\partial R}=\frac{1}{1+\partial_{r}^{2} S} \frac{\partial}{\partial r} \text {. }
$$

The divergence of the peculiar velocity with a Eulerian coordinate becomes a little complicated:

$$
\begin{aligned}
\nabla_{x} \cdot \mathbf{u} & =\frac{1}{R} \frac{\partial}{\partial R}\left(R \partial_{R} u\right) \\
& =\frac{1}{\left(r+\partial_{r} S\right)} \frac{1}{1+\partial_{r}^{2} S} \frac{\partial}{\partial r}\left\{\left(r+\partial_{r} S\right) \partial_{r} u\right\} .
\end{aligned}
$$

We decompose the Jacobian of the coordinate transformation to the order of the perturbation.

$$
J=1+J^{(1)}+J^{(2)}+J^{(3)},
$$

where

$$
\begin{gathered}
J^{(1)}=\partial_{i}^{2} S=\partial_{r}^{2} S+\frac{1}{r} \partial_{r} S, \\
J^{(2)}=\frac{1}{2}\left[\left(\partial_{i}^{2} S\right)\left(\partial_{j}^{2} S\right)-\left(\partial_{i} \partial_{j} S\right)\left(\partial_{j} \partial_{i} S\right)\right]=\frac{1}{r}\left(\partial_{r} S\right)\left(\partial_{r}^{2} S\right), \\
J^{(3)}=\operatorname{det}\left(\partial_{i} \partial_{j} S\right)=0 .
\end{gathered}
$$

Most of the above changes affect only higher-order approximation. In the EJN model, we must consider the change of Laplacian in Lagrangian space, i.e., 


$$
\nabla_{q}^{2} f(r)=\frac{1}{r} \frac{\partial}{\partial r}\left(r \frac{\partial f(r)}{\partial r}\right)=\frac{1}{r}\left(f^{\prime}(r)+r f^{\prime \prime}(r)\right),
$$

where the prime denotes the partial derivative with respect to $r$. Using the above deformations, we obtain the evolution equation for the EJN model with cylindrical symmetry. The linear perturbative equation for the EJN model is written as

$$
\begin{aligned}
\frac{1}{r} & \frac{\partial}{\partial r}\left[r\left\{\ddot{S}^{\prime}+2 \frac{\dot{a}}{a} \dot{S}^{\prime}-\frac{\kappa \gamma \rho_{b}^{\gamma-1}}{a^{2}} \frac{\partial}{\partial r}\left(\frac{1}{r} \frac{\partial}{\partial r}\left(r S^{\prime}\right)\right)\right\}\right] \\
& =-4 \pi G \rho_{b} \cdot \frac{1}{r} \frac{\partial}{\partial r}\left[r S^{\prime}\right] .
\end{aligned}
$$

When we choose an appropriate boundary condition, we can rewrite Eq. (A9) as

$$
\ddot{S}+2 \frac{\dot{a}}{a} \dot{S}-\frac{\kappa \gamma \rho_{b}^{\gamma-1}}{a^{2}}\left(S^{\prime \prime}+\frac{1}{r} S^{\prime}\right)=-4 \pi G \rho_{b} S .
$$

When we treat the EJN model in Cartesian coordinates, we can apply a Fourier transformation to the perturbative equations easily. However, with the cylindrical symmetry, because the fourth term on the left-hand side in Eq. (A10) derives convolution, even if we take only linear perturbation, we cannot obtain analytic solutions and we have to solve by numerical calculation.
[1] Ya. B. Zel'dovich, Astron. Astrophys. 5, 84 (1970).

[2] V.I. Arnol'd, S.F. Shandarin, and Ya. B. Zel'dovich, Geophys. Astrophys. Fluid Dyn. 20, 111 (1982).

[3] S. F. Shandarin and Ya. B. Zel'dovich, Rev. Mod. Phys. 61, 185 (1989).

[4] T. Buchert, Astron. Astrophys. 223, 9 (1989).

[5] T. Padmanabhan, Structure Formation in the Universe (Cambridge University Press, Cambridge, England, 1993).

[6] P. Coles and F. Lucchin, Cosmology: The Origin and Evolution of Cosmic Structure (Wiley, Chichester, 1995).

[7] V. Sahni and P. Coles, Phys. Rep. 262, 1 (1995).

[8] B. J. T. Jones, V. J. Martinez, E. Saar, and V. Trimble, Rev. Mod. Phys. 76, 1211 (2005).

[9] T. Tatekawa, astro-ph/0412025.

[10] T. Padmanabhan, gr-qc/0503107.

[11] D. Munshi, V. Sahni, and A. A. Starobinsky, Astrophys. J. 436, 517 (1994).

[12] V. Sahni and S. F. Shandarin, Mon. Not. R. Astron. Soc. 282, 641 (1996).

[13] A. Yoshisato, T. Matsubara, and M. Morikawa, Astrophys. J. 498, 48 (1998).

[14] A. Yoshisato, M. Morikawa, N. Gouda, and H. Mouri, astro-ph/0510107 [Astrophys. J. (to be published)].

[15] Ya. B. Zel'dovich, Sov. Astron. 26, 389 (1982).

[16] É. V. Kotok and S.F. Shandarin, Sov. Astron. 31, 600 (1987).

[17] S. N. Gurbatov, A. I. Saichev, and S. F. Shandarin, Mon. Not. R. Astron. Soc. 236, 385 (1989).

[18] J. M. Burgers, Proc. R. Neth. Acad. Sci. 43, 2 (1940).

[19] D. H. Weinberg and J. E. Gunn, Mon. Not. R. Astron. Soc. 247, 260 (1990).

[20] A. Nusser and A. Dekel, Astrophys. J. 362, 14 (1990).

[21] L. Kofman, D. Pogosyan, S.F. Shandarin, and A.L. Melott, Astrophys. J. 393, 437 (1992).
[22] A. L. Melott, S.F. Shandarin, and D.H. Weinberg, Astrophys. J. 428, 28 (1994).

[23] T. Buchert and A. Domínguez, Astron. Astrophys. 335, 395 (1998).

[24] J. Binney and S. Tremaine, Galactic Dynamics (Princeton University Press, Princeton, NJ, 1987).

[25] T. Buchert, A. Domínguez, and J. Perez-Mercader, Astron. Astrophys. 349, 343 (1999).

[26] T. Buchert and A. Domínguez, Astron. Astrophys. 438, 443 (2005).

[27] A. Domínguez, Phys. Rev. D 62, 103501 (2000).

[28] A. Domínguez, Mon. Not. R. Astron. Soc. 334, 435 (2002).

[29] S. Adler and T. Buchert, Astron. Astrophys. 343, 317 (1999).

[30] M. Morita and T. Tatekawa, Mon. Not. R. Astron. Soc. 328, 815 (2001).

[31] T. Tatekawa, M. Suda, K. I. Maeda, M. Morita, and H. Anzai, Phys. Rev. D 66, 064014 (2002).

[32] T. Tatekawa, Phys. Rev. D 71, 044024 (2005).

[33] T. Tatekawa, Phys. Rev. D 72, 024005 (2005).

[34] T. Tatekawa, Phys. Rev. D 69, 084020 (2004).

[35] S. A. Teukolsky, Phys. Rev. D 61, 087501 (2000).

[36] A.G. Doroshkevich, V.S. Ryaben'kin, and S.F. Shandarin, Astrofizica 9, 257 (1973).

[37] C. Hopf, Commun. Pure Appl. Math. 3, 201 (1950).

[38] J. D. Cole, Q. Appl. Math. 9, 226 (1951).

[39] S. Nerney, E. J. Schmahl, and Z. E. Musielak, Q. Appl. Math. 54, 63 (1996).

[40] W.H. Press, S. A. Teukolsky, W. T. Vetterling, and B.P. Flannery, Numerical Recipes in Fortran 77: The Art of Scientific Computing, 2nd Edition (Cambridge University Press, Cambridge, England, 1992). 